\title{
Non-invasive Monitoring of Bioreactors Using Atmospheric Pressure Chemical Ionization Mass Spectrometry
}

\author{
J. Langejuergen $^{1}$, M. Buering ${ }^{2}$, C. Wagner ${ }^{2}$, S. Beutel ${ }^{2}$, T. Scheper ${ }^{2}$, S. Zimmermann ${ }^{1}$ \\ ${ }^{1}$ Institute of Electrical Engineering and Measurement Technology, Department of Sensors and \\ Measurement Technology, Leibniz University Hannover, Hannover, Germany \\ Langejuergen@geml.uni-hannover.de \\ ${ }^{2}$ Institute of Technical Chemistry, Leibniz University Hannover, Hannover, Germany
}

\begin{abstract}
Generating biomass or biochemically active substances with constant and high quality and quantity requires continuous control of the biochemical environment within the used bioreactor. Thus, sensors or sensor systems that monitor the conditions during the cultivation are required. Since in most bioprocesses it is indispensable to prevent any contamination of the culture medium a continuously working sensor in these applications must either stand the process of sterilization or be non-invasive. In this work we present first results from a non-invasive analysis of the waste gas of a $2 \mathrm{~L}$ bioreactor during the cultivation of a model organism (E. coli strain K12) using a mass spectrometer with atmospheric pressure chemical ionization and a gas chromatographic pre-separation. Based on the used ionization method a detection of volatile organic compounds emitted during the lag-phase or the growth of the bacteria with extremely low detection limits is possible. We repeatedly observed various compounds with an abundance correlated to the growth of the culture which was monitored by further invasive and non-invasive sensors.
\end{abstract}

Key words: bioreactor, fermenter, waste gas analysis, mass spectrometry, atmospheric pressure chemical ionization.

\section{Introduction}

Generating biomass or biochemically active substances with constant and high quality and quantity in a bioreactor requires continuous control of the biochemical environment like temperature, $\mathrm{pH}, \mathrm{pO}_{2}$ or $\mathrm{pCO}_{2}$. Monitoring the nutrition concentration or the optical density of the culture provides information about the amount of biomass generated and the phase of growth. These invasive sensors must stand a sterilization process or require a sample of biomass to be taken during cultivation which increases the risk of contamination. Noninvasive sensors analyze the amount of consumed $\mathrm{O}_{2}$ or generated $\mathrm{CO}_{2}$ as well as in some applications $\mathrm{NH}_{3}, \mathrm{H}_{2}$ or $\mathrm{CH}_{4}$ in the waste gas. However, most of these sensors measure the abundance of rather unspecific compounds. Furthermore, photometric IR sensor systems are used [1] to analyze the abundance of primary or secondary metabolites. The sensitivity of photometric sensors is too low to ensure a reliable and fast control of the bioprocess especially in the early stage of cultivation. Thus, a non-invasive sensor system with sufficient selectivity and high sensitivity is necessary.

In this work we present first results of a noninvasive method for bioreactor monitoring using a mass spectrometer with atmospheric pressure chemical ionization (APCI) to analyze the waste gas of a $2 \mathrm{~L}$ bioreactor during the cultivation of $\mathrm{E}$. coli (K12). With this ionization method ultra-low detection limits for volatile organic compounds in the ppb even to ppt range can be achieved [2]. However, due to concurring ionization processes a preseparation using a gas chromatograph is still necessary.

Our objective is to recognize patterns of volatile organic compounds detectable when using APCI with a suitable GC separation that allow a reliable non-invasive evaluation of the vitality and productivity of the microorganism in bioreactors as shown for culture flasks in [3]. However, due to size and complexity of the used system we have not performed a direct sampling and an online evaluation of the waste gas yet. 
Thus, a medium term target is to use a compact and portable high resolution ion mobility spectrometer (IMS) [4] with the same ionization method and a similar pre-separation to monitor the cultivation of different microorganisms online.

\section{Method}

In this work 3 cultivations of the same microorganism (E. coli strain K12) grown under similar conditions are evaluated.

A $2 \mathrm{~L}$ bioreactor (Biostat Aplus, Satorius) filled with CgXII minimal growth medium with additional glucose $(8.3 \mathrm{~g} / \mathrm{L})$, ammonium sulfate $(15 \mathrm{~g} / \mathrm{L})$ and urea $(5 \mathrm{~g} / \mathrm{L})$ was used as shown in figure 1. The $\mathrm{pH}$ is kept constant at $\mathrm{pH}=6.8$ using 3 molar $\mathrm{NaOH}$ and 1 molar $\mathrm{HCl}$. Due to the fast growth of the used microorganisms no antibiotics were used. The gassing stirrer was supplied with about $2 \mathrm{~L} / \mathrm{min}$ sterile filtered air. The speed of the stirrer was controlled in order to obtain a constant $\mathrm{O}_{2}$ concentration in the medium. The temperature was kept constant at $37^{\circ} \mathrm{C}$.

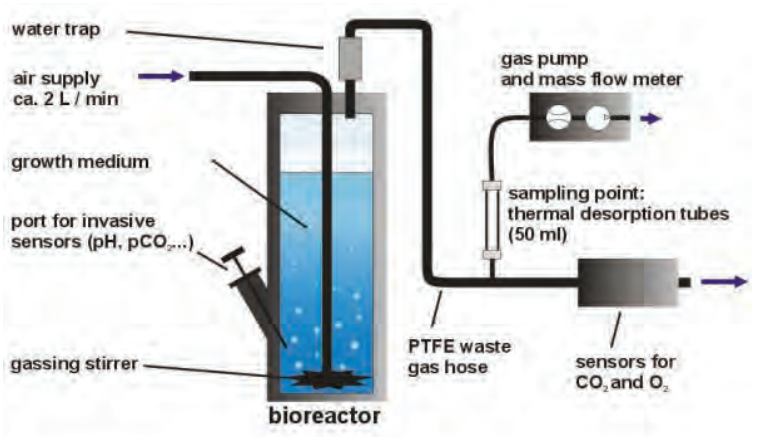

Fig. 1. Experimental setup of the bioreactor and the sampling point.

At $\mathrm{t}=0 \mathrm{~min}$ the growth medium was inoculated with the concentrated pre-culture $(9 \mathrm{ml}, \mathrm{OD} 600$ ca. 8 to 10). Every 2 to 3 hours a sample of the medium was taken and the optical density $(600 \mathrm{~nm})$ and the glucose concentration were analyzed. Furthermore, the $\mathrm{CO}_{2}$ and the $\mathrm{O}_{2}$ concentration of the waste gas were monitored as well as the $\mathrm{pCO}_{2}$ partial pressure within the medium and the amount of $\mathrm{NaOH}$ or $\mathrm{HCl}$ needed to stabilize the $\mathrm{pH}$ value.

At the sampling point 3 samples of $50 \mathrm{ml}$ waste gas were taken successively every 1 to 2 hours at a flow of $100 \mathrm{sccm}$. The sample gas flows through a thermal desorption tube $(6 \mathrm{~mm}$, glass filled with Tenax TA) where most of the volatile organic compounds adsorb. It is noteworthy to mention that in this case the thermal desorption tube is used for transportation purposes rather than for pre-concentration since a mass spectrometer with $\mathrm{APCl}$ is used. To obtain a comparable amount of detected compounds (not necessary the same compounds) with a conventional mass spectrometer with electron impact ionization (EI) several liters of sample had to be taken.

The sampled thermal desorption tubes with the adsorbed compounds from the waste gas were analyzed in an arbitrary order to avoid systematic errors. The experimental setup used for this analysis is shown in figure 2. The tubes were heated up with $7 \mathrm{~K} / \mathrm{min}$ to $180^{\circ} \mathrm{C}$ while $15 \mathrm{sccm}$ dry nitrogen flows through them (Gerstel TDS). At these temperatures all adsorbed volatile organic compounds desorb and are transferred via gas flow to the cryo trap (Gerstel $\mathrm{CIS}$ ) which is cooled at $-50^{\circ} \mathrm{C}$ by liquid nitrogen. After several minutes of trapping the cryo trap is heated with $12 \mathrm{~K} / \mathrm{min}$ to $200{ }^{\circ} \mathrm{C}$ to inject the trapped compounds into the gas chromatograph (GC).

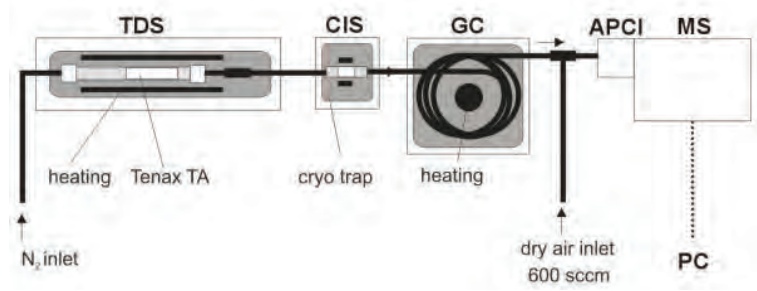

Fig. 2. Experimental setup for the analysis of the sampled thermal desorption tubes with APCI MS.

A GC column (Restek, RTX volatiles, $530 \mu \mathrm{m}$, $30 \mathrm{~m}$ ) is used with a carrier gas flow of $6 \mathrm{sccm}$ dry nitrogen. The starting temperature of the GC oven is $40{ }^{\circ} \mathrm{C}$ which is increased 2 minutes after the injection with a rate of $7 \mathrm{~K} / \mathrm{min}$ to $220^{\circ} \mathrm{C}$. After the pre-separation the compounds are ionized at atmospheric pressure by APCI as described in [5] using a radioactive $\beta^{-}$emitter (Tritium, $300 \mathrm{MBq}$ ). As carrier gas $600 \mathrm{sccm}$ of clean and dry air (dew point $-89^{\circ} \mathrm{C}$ ) is used. The ionized gas is transferred via carrier gas flow into the mass spectrometer and a mass spectrum of the positive ions is generated. Due to this soft ionization only few fragments are detected. Most positive ions are protonated $\left(\mathrm{M}+\mathrm{H}^{+}\right)$which means that $1 \mathrm{u}$ has to be added to the mass of the neutral molecule $\mathrm{M}$. At higher concentrations even dimer $\left(\mathrm{M}_{2} \mathrm{H}^{+}\right)$or trimer $\left(\mathrm{M}_{3} \mathrm{H}^{+}\right)$ions can be present in the spectrum.

\section{Results}

In figure 3 the glucose concentration and the optical density within the bioreactor during one cultivation are shown. During the first 4 hours after inoculation only a small increase in optical density and a minor decrease in glucose concentration are visible. This is caused by the adaption of the microorganisms to the biochemical environment within the reactor (lag- 
phase). After 5 to 6 hours an exponential decrease in glucose concentration and a corresponding increase in optical density were detected due to the exponential cell growth. After 10 hours the glucose is digested and no further growth can be observed. At this point most microorganisms die due to a lack of nutrients. This is also visible in figure 4 where the $\mathrm{CO}_{2}$ concentration of the waste gas is shown. During the first hours of cultivation only a small increase in emitted $\mathrm{CO}_{2}$ was recorded while during the exponential growth phase the amount of emitted $\mathrm{CO}_{2}$ increases exponentially. After approximately 10 hours the $\mathrm{CO}_{2}$ concentration drops almost to its initial level due to the death of most microorganisms.

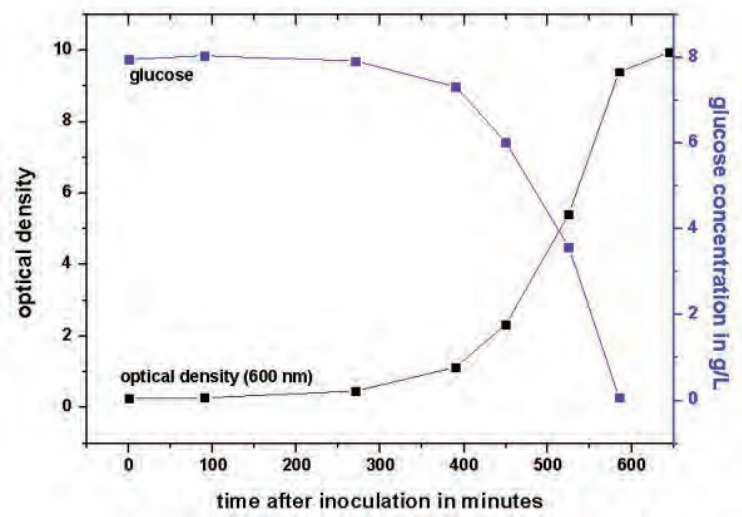

Fig. 3. Optical density at $600 \mathrm{~nm}$ and glucose concentration.

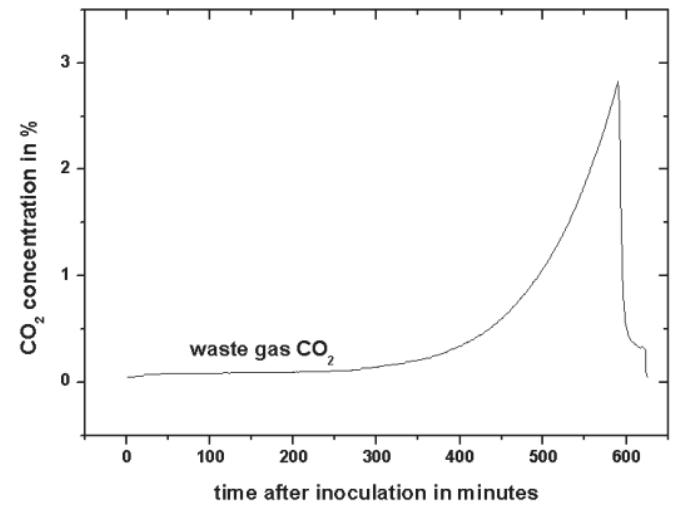

Fig. 4. Waste gas $\mathrm{CO}_{2}$ concentration.

The results from the GC-MS analysis of the waste gas samples are presented as heat map as shown in figure 5 . The GC retention time is plotted at the ordinate and the mass $(\mathrm{m} / \mathrm{z})$ at the abscissa. The signal intensity of a certain mass at a certain retention time is represented by color.

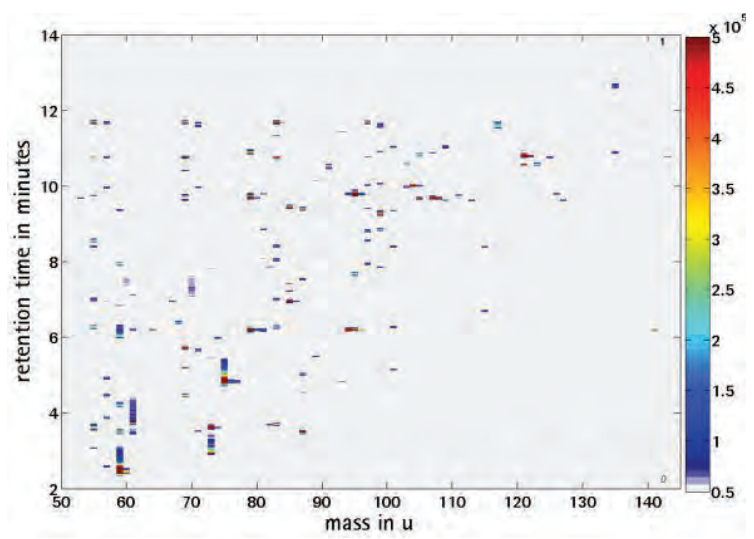

Fig. 5 Results of the GC-MS analysis of one thermal desorption tube.

Depending on the chosen threshold about hundred compounds are significantly present in a $50 \mathrm{ml}$ sample of waste gas. Nevertheless, since most GC-MS data libraries refer to default mass spectra generated with El most of these compounds are not identified yet. Since APCl is a rather soft ionization method most compounds only show few fragments.

The heat maps were evaluated in order to recognize certain compounds related to the glucose concentration or the amount of biomass present in the reactor at the time of waste gas extraction. 10 compounds were found that show a significant correlation to amount of biomass and have comparable trends in signal intensity in all 3 cultivations. Two of them are displayed here in detail.

In figure 6 the logarithmic signal intensity of a compound at a GC retention time of $6.18 \mathrm{~min}$ is shown. The corresponding APCI mass spectrum with a base peak of $94 \mathrm{u}$ is illustrated in figure 7. A significant increase in signal intensity is already visible even before an exponential growth begins. Since the abundance of this substance reaches a maximum when most of the culture already died from a lack of nutrients it is not likely to be a metabolite of a healthy $\mathrm{E}$. coli culture but rather a possible marker for dead or dying microorganisms. Nevertheless, a possible high solubility of this or any other compound in the grow media might cause a delay between the generation and the detection of a substance and must always be considered when interpreting the compounds present in the waste gas. 


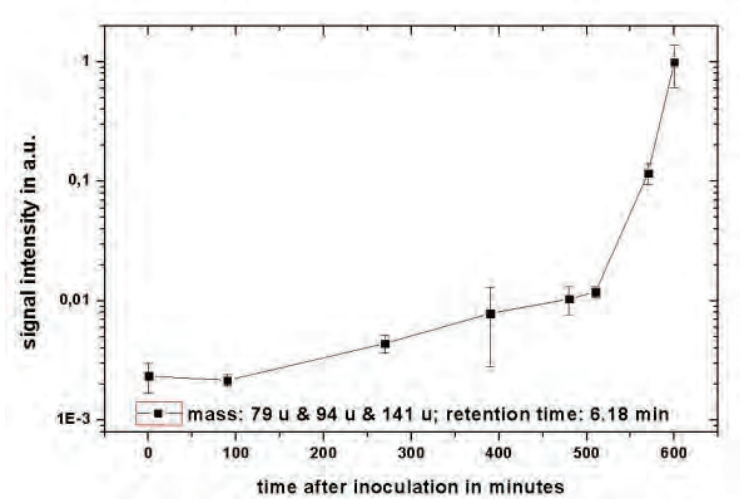

Fig. 6. Change in signal intensity for a compound at a retention time of $6.18 \mathrm{~min}$.

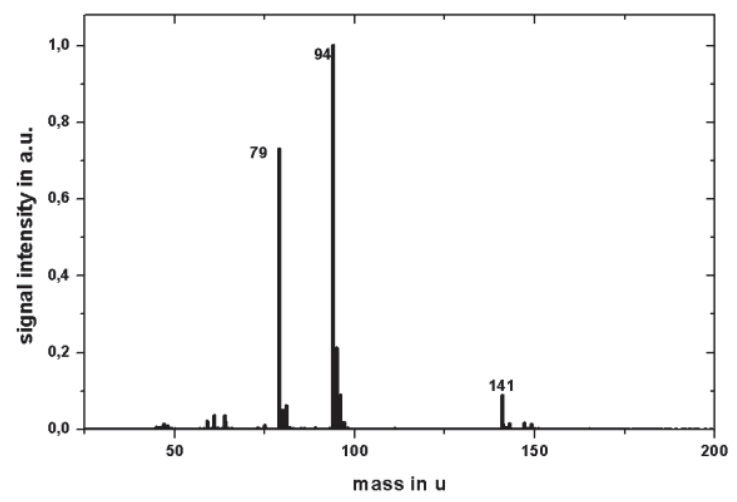

Fig. 7. APCI mass spectrum at a retention time of $6.18 \mathrm{~min}$

In figure 8 the decreasing signal intensity of a compound at a $\mathrm{GC}$ retention time of $2.85 \mathrm{~min}$ is shown. The corresponding $\mathrm{APCl}$ mass spectrum with a base peak of $75 \mathrm{u}$ is illustrated in figure 9. This compound decreases faster than the glucose concentration within the growth media.

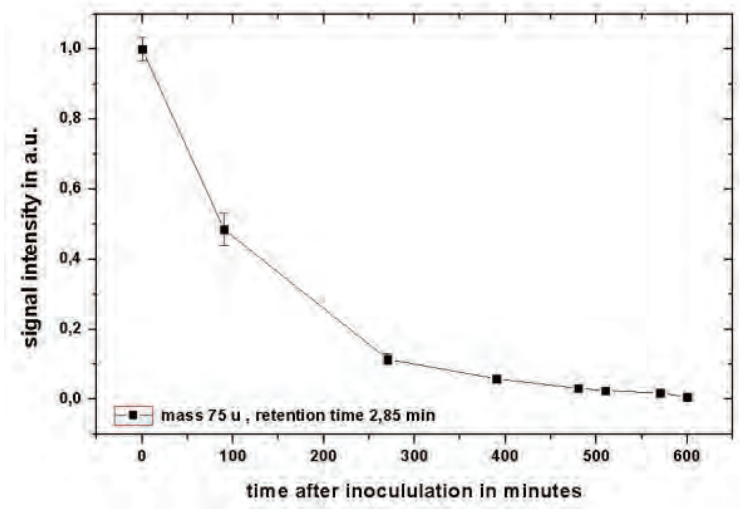

Fig. 8. Change in signal intensity for a compound at a retention time of $2.85 \mathrm{~min}$.

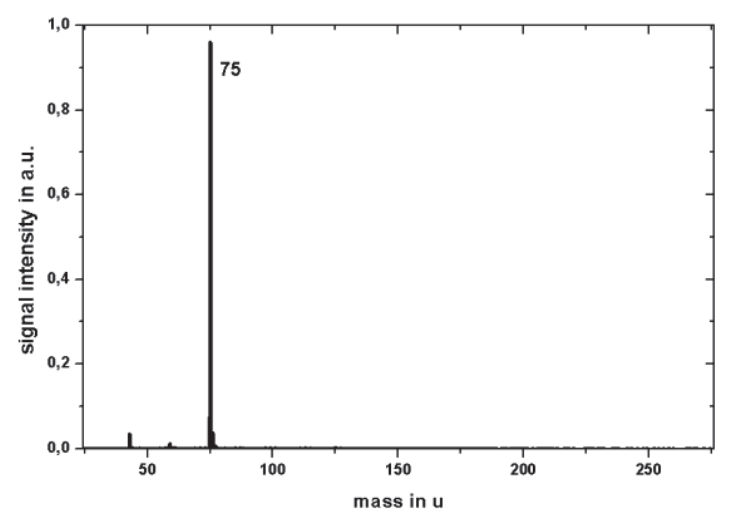

Fig. 9. APCl mass spectrum at a retention time of $2.85 \mathrm{~min}$.

\section{Conclusion}

Using APCI-MS with GC pre-separation it is possible to recognize several substances present in the waste gas of a bioreactor that are correlated to the growth of the model organism (E. coli strain K12). Some of these compounds show a decrease others an increase over several orders of magnitude in signal intensity during the cultivation.

A medium term target is to use a compact and portable high resolution ion mobility spectrometer (IMS) with the same ionization method and a similar pre-separation to monitor the cultivation of different microorganisms online.

\section{References}

[1] D. Landgrebe, C. Haake, T. Höpfner, S. Beutel, B. Hitzmann, T. Scheper, M. Rhiel, K. F. Reardon: "On-line infrared spectroscopy for bioprocess monitoring" , Appl. Microbiol Biotechnol (2010); doi: 10.1007/s00253-0102743-8

[2] J. Langejuergen, P. Cochems, S. Zimmermann: „A simple atmospheric pressure ionization source coupled to a time-of-flight mass spectrometer for breath analysis", Proceedings, 10. Sensor Symposium Dresden, pp. 87-90, (2011); doi: 10.5162/10dss2011/5.1

[3] N. Kunze, J. Göpel, M. Jünger, M. Quintel, T. Perl: „Detection and validation of volatile metabolic patterns over different strains of two human pathogenic bacteria during their growth in a complex medium using multi-capillary columnion mobility spectrometry (MCC-IMS)"Appl. Microbiol. Biotechnolo. (2013) 97:3665-3676; doi: $10.1007 / \mathrm{s} 00253-013-4762-8$

[4] A. Kirk, M. Allers, P. Cochems, J. Langejuergen, S. Zimmermann," A compact high resolution ion mobility spectrometer for fast trace gas analysis" Analyst, vol. 138,18 , pp. 5200-5207,(2013) doi: 10.1039/C3AN00231D

[5] G. A. Eiceman, Z. Karpas, „Ion Mobility Spectrometry" (2010) CRC Press 